\title{
Optical Flow Computation with Fourth Order Partial Differential Equations
}

\author{
Xiaoxin Guo, Zhiwen Xu, Yueping Feng, Yunxiao Wang, and Zhengxuan Wang \\ Key Laboratory of Symbol Computation and Knowledge Engineering of the Ministry \\ of Education, College of Computer Science and Technology, Jilin University, \\ Qianjin Street 2699\#, Changchun, 130012, P.R. China \\ xiaoxin@mail.jl.cn, guoxx@jlu.edu.cn
}

\begin{abstract}
In this paper, we propose a new hybrid optical flow computation with fourth order partial differential equations (PDEs). The integration of local and global optical flow methods exploits fourth order PDEs rather than second order for the purpose of the improvement of smoothness and accuracy of the estimated optical flow field. Furthermore, we describe the implementation of the method in detail. The experiments show that the employment of fourth order PDEs benefits the improvement of the two aspects of the resulting optical flow field.
\end{abstract}

\section{Introduction}

Optical flow is the term used to indicate the distribution of velocity generated by the relative motion between an object and the camera, over the points of an image sequence, and carries important information which is valuable for analyzing dynamic scenes or motion in video. Optical flow is determined by the velocity vector of each pixel in each frame. One of the most appealing features of optical flow computation methods is perhaps their generality, which provides a basis for their application in a broad spectrum of computer vision applications. Several schemes have been devised for calculating optical flow based on two or more frames of a sequence. These schemes can be classified into two general categories: local methods, which may optimize some local energy-like expression, and global strategies, which attempt to minimize a global energy functional.

There exists a very large number of publications on optical flow computation [1][2]. Schnörr [3] sketched a framework for supplementing global energy functionals with multiple equations that provide local data constraints. He suggested to use the output of Gaussian filters shifted in frequency space [4] or local methods incorporating second-order derivatives [4][5], but did not consider methods of Lucas-Kanade type.

While the noise sensitivity of local differential methods has been studied intensively in recent years [6]-[11], the noise sensitivity of global differential methods has been analyzed to a significantly smaller extent. In this context, Galvin et al. [12] have compared a number of classical methods where small amounts of Gaussian noise had been added. Their conclusion was similar to the findings of Barron et al. [13]: the 
global approach of Horn and Schunck is more sensitive to noise than the local LucasKanade method.

This paper proposes a new hybrid optical flow computation, which incorporates global strategies into local methods. Different from other optical flow computations, our work uses fourth order partial differential equations (PDEs) instead of second order PDEs as a technique to avoid over-smooth effects while achieving good tradeoff between smoothness and faithfulness to the data. Because of the use of nonlinear penalty function, the optical flow computation can also achieves the goal of preserving discontinuity of optical flow field. In implementation, we adopt the method called successive overrelaxation (SOR) [17] to numerically approximate the optical flow equation with fourth order PDEs. The iterative method possesses good properties in both temporal and spatial computation complexity. The experimental results show the validity and applicability of the proposed method.

The paper is organized as follows. Section 2 presents optical flow computation including Lucas-Kanade and Horn-Schunck methods, and Section 3 proposes our method, involving basic ideas and model. We describe the implementation of the proposed method in Section 4. Experiments are presented in Section 5 and the paper is concluded in Section 6.

\section{Optical Flow Computation}

Consider an image sequence $g(x, y, t)$, where $(x, y)$ denotes the location within a rectangular image domain $\Omega$, and $t \in[0, T]$ denotes time. Many differential methods for optical flow are based on the assumption that the grey values of image objects in subsequent frames do not change over time:

$$
g_{x} u+g_{y} v+g_{t}=0,
$$

where the displacement field $(u, v)$ is called optical flow, and subscripts denote partial derivatives.

Evidently, this single equation is not sufficient to uniquely compute the two unknowns $u$ and $v$ (aperture problem): In order to cope with the aperture problem, Lucas et al. [14][15] proposed to assume that the unknown optical flow vector is constant within some neighborhood of size $\rho$. In this case it is possible to determine the two constants $u$ and $v$ at some location $(x, y, t)$ from a weighted least square fit by minimizing the function

$$
E_{L K}(u, v)=K_{\rho} *\left(\left(g_{x} u+g_{y} v+g_{t}\right)^{2}\right),
$$

where $K_{\rho}$ is a Gaussian kernel with the standard deviation $\rho$, and $*$ is a convolution operator. The method is robust against noise. However, it constitutes the most severe drawback of local gradient methods: its flow fields are nondense.

In order to end up with dense flow estimates one may embed the optical flow constraint into a regularization framework. Horn et al. [16] have pioneered this class of global differential methods. They determine the unknown functions $u(x, y, t)$ and $v(x, y, t)$ as the minimizers of the global energy functional 


$$
E_{H S}(u, v)=\int_{\Omega}\left(\left(g_{x} u+g_{y} v+g_{t}\right)^{2}+\alpha\left(|\nabla u|^{2}+|\nabla v|^{2}\right)\right) d x d y
$$

where the smoothness weight $\alpha>0$ serves as a regularization parameter, and $\nabla$ is a gradient operator. The use of the regularizer results in dense flow fields and makes subsequent interpolation steps obsolete. This is a clear advantage over local methods. Unfortunately, the method is sensitive to noise.

Since both local and global differential methods have complementary advantages and shortcomings, it would be interesting to construct a hybrid technique that constitutes the beneficial factors of two methods: It should combine the robustness of local methods with the density of global approaches.

An existing hybrid method employs the convolution kernel with standard deviation $\rho$ for local methods and the optical flow constraint for global approaches. The estimated optical flow field is the solution of the minimization problem, given by the following functional

$$
E(u, v)=\int_{\Omega}\left(K_{\rho} *\left(g_{x} u+g_{y} v+g_{t}\right)^{2}+\alpha(\psi(|\nabla u|)+\psi(|\nabla v|))\right) d x d y,
$$

where $\psi(\cdot)$ is called a potential function. When $\rho=0, \psi(\cdot)=(\cdot)^{2}$, the above equation will be reduced to Eq. (3).

\section{Smoothness Constraints Using Fourth Order PDEs}

Although these techniques using second order PDEs as smoothness constraints are able to achieve a good tradeoff between smoothness and optical flow constraints, they tend to cause over-smooth effect as a result of the fact that second order PDEs are strong constraints. This result is undesirable and is likely to cause a computer vision system to falsely recognize the motions of different object as ones belong to the same object.

This over-smooth effect is, to a large extent, inherent in the nature of second order PDEs. Since second order derivatives are zero only if the optical flow field is linearmonotonously changing, these PDEs for the two velocity components will evolve toward and settle down to an optical flow field with constant gradients if the field is infinite. For fields of limited support, however, symmetric boundary condition is usually employed in order to avoid motion distortion at the boundaries. Then these PDEs will evolve toward a constant field. Since these PDEs are usually designed such that optical flows in smooth areas evolve faster than those around rough areas in order to preserve discontinuity while removing noise, consistent flow areas will become flat faster than less consistent areas. Consequently, the optical flow field is likely to evolve at early stage into such a vector field that may be approximated by constant subfields. The boundaries of these subfields may coincide with true segmentations of the moving objects, but may result in incorrect motion estimations due to the oversmooth effect.

For this, we propose a novel smoothness constraints using fourth order PDEs for optical flow equations, forming a new hybrid method. First consider the following functional defined in the space of continuously varying vectors over a support of $\Omega$ : 


$$
E(u, v)=\int_{\Omega}\left(K_{\rho} *\left(g_{x} u+g_{y} v+g_{t}\right)^{2}+\alpha(\psi(|\Delta u|)+\psi(|\Delta v|))\right) d x d y,
$$

where $\Delta$ is a Laplacian operator. Different from Eq.(4), the smoothness term in Eq. (5) use Laplacian operators $\psi(|\Delta u|)+\psi(|\Delta v|)$ rather than gradient operators $\psi(|\nabla u|)+\psi(|\nabla v|)$. We require that the potential function $\psi(\cdot) \geq 0$ and is an increasing function:

$$
\psi^{\prime}(\cdot)>0
$$

so that the functional is an increasing function with respect to the smoothness of the field as measured by $|\Delta u|$ and $|\Delta v|$. Therefore, the minimization of the functional is equivalent to smoothing the optical flow field. The use of the potential function enables the construction of nonlinear equations. For example, $\psi(\cdot)$ can adopt a Huber function as a penalty function to control the discontinuity in the a priori model. The minimum of the functional may be found by solving the following Euler's equation for all $(x, y) \in \Omega$,

$$
\begin{aligned}
& \Delta\left[\psi^{\prime}(|\Delta u|) \Delta u /|\Delta u|\right]-K_{\rho} * \alpha^{-1}\left(g_{x} g_{x} u+g_{x} g_{y} v+g_{x} g_{t}\right)=0, \\
& \Delta\left[\psi^{\prime}(|\Delta v|) \Delta v /|\Delta v|\right]-K_{\rho} * \alpha^{-1}\left(g_{y} g_{x} u+g_{y} g_{y} v+g_{y} g_{t}\right)=0 .
\end{aligned}
$$

An optical flow field whose velocity components both satisfy a plane equation refers to a plane optical flow field. For its velocity components, their Laplacians are zero, so they satisfy Eq. (5). Therefore, a plane field is obviously a global minimum of the functional (5).

Let $\Omega_{i}, i=1,2, \ldots, n$ be a partition of $\Omega$. For an approximated optical flow composed of plane subfields, we require that the plane subfields be such that the combined field is continuous. Therefore, the velocity components in any two adjacent subfields must be on different planes; otherwise, we can combine them as one. Let us denote $\partial \Omega_{i}$ as the boundary of portion $\Omega_{i}$, then $\Omega_{i}-\partial \Omega_{i}$ is the interior of $\Omega_{i}$. It is obvious that

$$
\nabla u_{i}(x, y)=c_{1}, \nabla v_{i}(x, y)=c_{2},(x, y) \in\left(\Omega_{i}-\partial \Omega_{i}\right),
$$

where $c_{1}$ and $c_{2}$ are both constant. So we have

$$
\Delta u_{i}(x, y)=0, \Delta v_{i}(x, y)=0,(x, y) \in\left(\Omega_{i}-\partial \Omega_{i}\right),
$$

for $i=1,2, \ldots, n$. Therefore,

$$
\Delta u(x, y)=0, \Delta v(x, y)=0,(x, y) \in(\Omega-\partial \Omega),
$$

where $\partial \Omega=\cup_{i=1}^{n} \partial \Omega_{i}$. Since it is required that the velocity components in any two adjacent subfields be on different planes, we have

$$
\nabla u_{i} \neq \nabla u_{j}, \text { or } \nabla v_{i} \neq \nabla v_{j}
$$


for any two adjacent portions $\Omega_{i}$ and $\Omega_{j}$. This indicates that the gradient for the components is not continuous at the boundary $\partial \Omega$. So we have

$$
\Delta u(x, y)=\infty \text {, or } \Delta v(x, y)=\infty .
$$

If we require that

$$
\psi^{\prime}(\infty)=0
$$

we then have

$$
\psi^{\prime}(|\Delta u|) \Delta u /|\Delta u|=0, \psi^{\prime}(|\Delta v|) \Delta v /|\Delta v|=0,
$$

for all $(x, y) \in \Omega$. Therefore, an optical flow field composed of plane subfields satisfies the Euler's equation.

\section{Implementation}

The differential equation (7) and (8) may be solved numerically using an iterative SOR method [17]. The SOR method is a good compromise between simplicity and efficiency. Assuming a space grid size of $h$, we discretize the space coordinates as follows:

$$
x=i h, y=j h, i=1,2, \ldots, N, j=1,2, \ldots, N,
$$

where $N h \times N h$ is the size of image support. We then employ a three-stage approach to calculate the constraint terms of Eq. (7) and (8). At the first stage, we calculate the Laplacians of the optical flow vector functions as

$$
\begin{gathered}
\Delta u_{i, j}^{k}=\left(u_{i+1, j}^{k}+u_{i-1, j}^{k}+u_{i, j+1}^{k}+u_{i, j-1}^{k}-4 u_{i, j}^{k}\right) / h^{2}, \\
\Delta v_{i, j}^{k}=\left(v_{i+1, j}^{k}+v_{i-1, j}^{k}+v_{i, j+1}^{k}+v_{i, j-1}^{k}-4 v_{i, j}^{k}\right) / h^{2},
\end{gathered}
$$

with symmetric boundary conditions:

$$
\begin{aligned}
& u_{i,-1}^{k}=u_{i, 0}^{k}, u_{i, J+1}^{k}=u_{i, J}^{k}, v_{i,-1}^{k}=v_{i, 0}^{k}, v_{i, J+1}^{k}=v_{i, J}^{k}, i=1,2, \ldots, N ; \\
& u_{-1, j}^{k}=u_{0, j}^{k}, u_{I+1, j}^{k}=u_{I, j}^{k}, v_{-1, j}^{k}=v_{0, j}^{k}, v_{I+1, j}^{k}=v_{I, j}^{k}, j=1,2, \ldots, N .
\end{aligned}
$$

At the second stage, we calculate the value of the following functions

$$
\varphi(\Delta u)=\psi^{\prime}(|\Delta u|) \Delta u /|\Delta u| \text { and } \varphi(\Delta v)=\psi^{\prime}(|\Delta v|) \Delta v /|\Delta v|
$$

For convenience, the above equations can be discretized as

$$
\varphi_{u(i, j)}^{k}=\varphi\left(\Delta u_{(i, j)}^{k}\right) \text { and } \varphi_{v(i, j)}^{k}=\varphi\left(\Delta v_{(i, j)}^{k}\right) .
$$

Finally, the numerical approximation to the differential equation (7) and (8) is given as 


$$
\begin{aligned}
& u_{(i, j)}^{k+1}=(1-\omega) \varphi_{u(i, j)}^{k}+\omega\left[\sum_{(p, q) \mathcal{N}^{-}(i, j)} \varphi_{u(p, q)}^{k+1}+\sum_{(p, q) \mathcal{N}^{+}(i, j)} \varphi_{u(p, q)}^{k}\right. \\
& \left.-\frac{h^{2}}{\alpha}\left(g_{x}(i, j) g_{y}(i, j) v_{(i, j)}^{k}+g_{x}(i, j) g_{t}(i, j)\right)\right] /\left[|\mathcal{N}(i, j)|+\frac{h^{2}}{\alpha} g_{x}(i, j) g_{x}(i, j)\right], \\
& v_{(i, j)}^{k+1}=(1-\omega) \varphi_{v(i, j)}^{k}+\omega\left[\sum_{(p, q) \mathcal{N}^{-(i, j)}} \varphi_{v(p, q)}^{k+1}+\sum_{(p, q) N^{+}(i, j)} \varphi_{v(p, q)}^{k}\right. \\
& \left.-\frac{h^{2}}{\alpha}\left(g_{y}(i, j) g_{x}(i, j) u_{(i, j)}^{k+1}+g_{y}(i, j) g_{t}(i, j)\right)\right] /\left[|\mathcal{N}(i, j)|+\frac{h^{2}}{\alpha} g_{y}(i, j) g_{y}(i, j)\right],
\end{aligned}
$$

with symmetric boundary conditions

$$
\begin{aligned}
& \varphi_{u(i,-1)}^{k}=\varphi_{u(i, 0)}^{k}, \varphi_{u(i, J+1)}^{k}=\varphi_{u(i, J)}^{k}, \varphi_{v(i,-1)}^{k}=\varphi_{v(i, 0)}^{k}, \varphi_{v(i, J+1)}^{k}=\varphi_{v(i, J)}^{k}, i=1,2, \ldots, N ; \\
& \varphi_{u(-1, j)}^{k}=\varphi_{u(0, j)}^{k}, \varphi_{u(I+1, j)}^{k}=\varphi_{u(I, j)}^{k}, \varphi_{v(-1, j)}^{k}=\varphi_{v(0, j)}^{k}, \varphi_{v(I+1, j)}^{k}=\varphi_{v(I, j)}^{k}, j=1,2, \ldots, N ;
\end{aligned}
$$

where $|\mathcal{N}(i)|$ denotes the number of neighborhoods of pixel $(i, j)$, and

$$
\begin{aligned}
& \mathcal{N}^{-}(i, j)=\{(p, q) \in \mathcal{N}(i, j) \mid q<j \text { or } q=j, p<i\}, \\
& \mathcal{N}^{+}(i, j)=\{(p, q) \in \mathcal{N}(i, j) \mid q>j \text { or } q=j, p>i\} .
\end{aligned}
$$

\section{Experiments}

We now demonstrate the performance of the proposed optical flow computation using fourth order PDE. We use our scheme to computer optical flow and compare the results with those processed using second order PDE. For both PDEs we use the following function

$$
\psi^{\prime}(s)=s /\left(1+(s / \kappa)^{2}\right),
$$

with $\kappa=1$. Obviously, the above equation satisfies Eq. (6) and (14). Without its prototype function $\psi(s)$, we may directly use the iterative approach presented in Section 5. Besides, the experiment uses the following parameters $\alpha=950, \rho=4.55$ and $h=1$.

We use two "Lena" images (Fig. 1 (a) and (b)) as original images between which the misalignment ( 2 degrees rotation) exists. Since the true optical flow field (Fig. 1 (e)) is known, it is convenient to conduct the experiments for quantitative comparison. Fig. 1 (c) and (d) are the two original images contaminated by Gaussian noise with the deviation $\sigma_{n}^{2}=0.006$. Two different optical flow computations, using second and fourth order PDE, respectively, are operated on the two degraded images. The results are shown in Fig. 1 (f) and (g). From the results, we see that the estimated flow fields are consistent with the true one.

In order to quantitatively compare the two schemes, we give for different noise levels the average angular errors, shown in Table 1, computed by

$$
\arccos \left(\left(u_{c} u_{e}+v_{c} v_{e}+1\right) / \sqrt{\left(u_{c}^{2}+v_{c}^{2}+1\right)\left(u_{e}^{2}+v_{e}^{2}+1\right)}\right),
$$

where $\left(u_{c}, v_{c}\right)$ denotes the correct flow, and $\left(u_{e}, v_{e}\right)$ is the estimated flow[13]. 


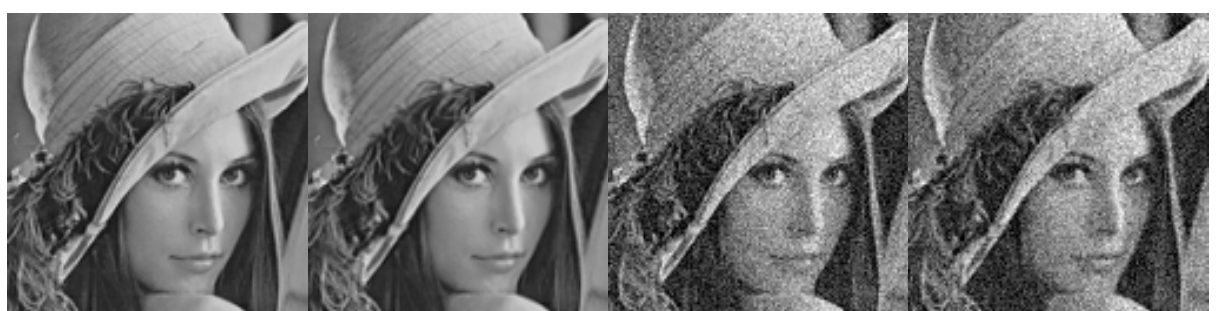

(a)

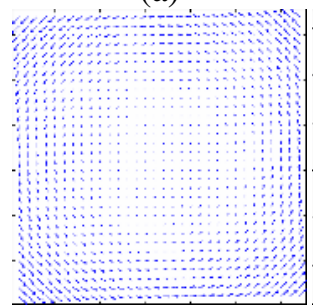

(e) (b)

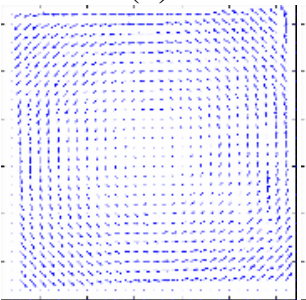

(f) (c)

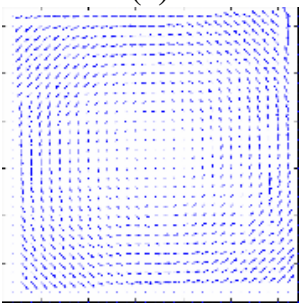

(g)

(d)

Fig. 1. Hybrid optical flow computations. (a) original image (frame 1); (b) original image (frame 2); (c) noisy image with Gaussian noise $\sigma_{n}^{2}=0.006$ (frame 1); (d) noisy image with Gaussian noise $\sigma_{n}^{2}=0.006$ (frame 2); (e) the true optical flow field; (f) the estimated optical flow field using 2nd order PDEs; (g) the estimated optical flow field using 4th order PDEs.

Table 1. Average angular errors computed with varying standard deviations $\sigma_{n}^{2}$ of Gaussian noise

\begin{tabular}{c|c|c}
\hline$\sigma_{n}^{2}$ & $2^{\text {nd }}$ order PDEs & $4^{\text {th }}$ order PDEs \\
\hline 0 & $2.632^{\circ}$ & $1.705^{\circ}$ \\
\hline 0.0015 & $3.387^{\circ}$ & $3.100^{\circ}$ \\
\hline 0.006 & $4.924^{\circ}$ & $4.609^{\circ}$ \\
\hline
\end{tabular}

For the hybrid methods, the influence of constraints using second and fourth order PDEs, respectively, on the resulting flow field is different. For low noise levels, the accuracy for the latter is higher than that for the former. This indicates the usefulness of filling-in effect. For high noise levels, on the other hand, the latter can rival the former for robustness. Moreover, the hybrid method using fourth order PDEs doesn't reduce necessary smoothing.

\section{Conclusions}

In general, the optical flow computation with fourth order PDEs consider the two aspects: accuracy, which relies on the filling-in effect in flat areas, and robustness, which enhances the ability to resist noise. In addition, small angular errors show the ability to preserve the discontinuity of the flow field using fourth order PDEs. 
Therefore, compared with the method with second order PDEs, the proposed method is superior to the former.

\section{References}

1. Mitiche, A., Bouthemy, P.: Computation and analysis of image motion: A synopsis of current problems and methods. International Journal of Computer Vision, (1996) 19(1):29-55

2. Stiller, C., Konrad, J.: Estimating motion in image sequences. IEEE Signal Processing Magazine, (1999) 16:70-91

3. Schnörr, C.: On functionals with greyvalue-controlled smoothness terms for determining optical flow. IEEE Transactions on Pattern Analysis and Machine Intelligence, (1993) 15:1074-1079

4. Fleet, D.J., Jepson, A.D.: Computation of component image velocity from local phase information. International Journal of Computer Vision, (1990) 5(1):77-104

5. Tretiak, Pastor, L.: Velocity estimation from image sequences with second order differential operators. In Proc. Seventh International Conference on Pattern Recognition, Montreal, Canada, (1984) 16-19

6. Uras, S., Girosi, F., Verri, A., V. Torre, A.: Computational approach to motion perception. Biological Cybernetics, (1988) 60:79-87

7. Bainbridge-Smith, Lane, R.G.: Determining optical flow using a differential method. Image and Vision Computing, (1997) 15(1):11-22

8. Fermüller, Shulman, D., Aloimonos, Y.: The statistics of optical flow. Computer Vision and Image Understanding, (2001) 82(1):1-32

9. Jähne: Digitale Bildverarbeitung. Springer: Berlin. (2001)

10. Ohta, N.: Uncertainty models of the gradient constraint for optical flow computation. IEICE Transactions on Information and Systems, (1996) E79-D(7):958-962

11. Simoncelli, E.P., Adelson, E.H., Heeger, D.J.: Probability distributions of optical flow. In Proc. 1991 IEEE Computer Society Conference on Computer Vision and Pattern Recognition, IEEE Computer Society Press: Maui, HI, (1991) 310-315

12. Galvin, B., McCane, B., Novins, K., Mason, D., Mills, S.: Recovering motion fields: An analysis of eight optical flow algorithms. In Proc. 1998 British Machine Vision Conference, Southampton, England. (1998)

13. Barron, J. L., Fleet, D. J., Beauchemin, S. S.: Performance of optical flowtechniques. International Journal of Computer Vision, (1994) 12(1):43-77

14. Lucas, B., Kanade, T.: An iterative image registration technique with an application to stereo vision. In: Proc. Seventh International Joint Conference on Artificial Intelligence, Vancouver, Canada, (1981) 674-679

15. Lucas, B. D.: Generalized image matching by the method of differences. PhD thesis, School of Computer Science, Carnegie-Mellon University, Pittsburgh, PA. (1984)

16. Horn, K. P., Schunk, B. G.: Determining optical flow. Aritificial Intelligence, (1981) 17:185-203

17. Young, D. M.: Iterative Solution of Large Linear Systems. Academic Press: New York. (1971) 